\title{
A case of ARDS due to adenoviral pneumonia
}

\begin{abstract}
The acute respiratory distress syndrome (ARDS) is characterized by a non-cardiogenic pulmonary edema with bilateral chest radiograph opacities and hypoxemia refractory to oxygen therapy.Adenovirus is a frequent cause of mild self-limiting upper respiratory tract infection, gastroenteritis, and conjunctivitis in infants and young children. Fatal infections (severe pneumonia progressing to respiratory failure, septic shock and/or encephalitis) are rare among immunocompetent adults. We report a case of a twenty-two years old pregnant woman with severe adenovirus pneumonia who has presented with sudden onset of respiratory distress, progressed to respiratory failure rapidly and has made a successful recovery with supportive measures.
\end{abstract}

Keywords: Acute respiratory distress syndrome (ARDS), adenovirus, positive end -expiratory pressure (PEEP), high-flow oxygen nasal cannulae (HFNC)
Case Report

Volume 8 Issue I - 2018

\author{
Fatma Irem Yesiler,' Mehmet Akif Izgi, ${ }^{2}$ Akif \\ Demirel, ${ }^{3}$ Serdar Ozdemir ${ }^{3}$ \\ 'Department of Intensive Care Unit, Sanliurfa Training and \\ Research Hospital,Turkey \\ ${ }^{2}$ Department of Internal Medicine, Sanliurfa Training and \\ Research Hospital,Turkey \\ ${ }^{3}$ Department of Anesthesiology, Sanliurfa Training and Research \\ Hospital,Turkey
}

\begin{abstract}
Correspondence: Fatma Irem Yesiler, Department of Intensive Care Unit, Sanliurfa Training and Research Hospital, 63250 Sanliurfa, Turkey, Tel +90 4l4 317|823, Fax +90 414317 262I, Email fatmairem84@hotmail.com
\end{abstract}

Received: January 25, 2018 | Published: February 26, 2018

\section{Introduction}

The acute respiratory distress syndrome (ARDS) is characterized by a non-cardiogenic pulmonary edema with bilateral chest radiograph opacities and hypoxemia refractory to oxygen therapy. ARDS is an acute, diffuse, inflammatory lung injury that leads to increased pulmonary vascular permeability, increased lung weight, and a loss of aerated tissue. The pathological hallmark is diffuse alveolar damage (ie, alveolar edema with or without focal hemorrhage, acute inflammation of the alveolar walls, and hyaline membranes). It is a common cause of admission to the intensive care unit (ICU) due to hypoxemic respiratory failure requiring mechanical ventilation. The severity of the hypoxemia defines the severity of the ARDS. Mild ARDS exists when the $\mathrm{PaO}_{2} / \mathrm{FiO}_{2}$ is $>200 \mathrm{mmHg}$, but $\leq 300 \mathrm{mmHg}$, on invasive or non-invasive ventilator settings that include a positive end-expiratory pressure (PEEP) or continuous positive airway pressure (CPAP) $\geq 5$ $\mathrm{cm} \mathrm{H}_{2} \mathrm{O}$. Moderate ARDS exists when the $\mathrm{PaO}_{2} / \mathrm{FiO}_{2}$ is $>100 \mathrm{mmHg}$, but $\leq 200 \mathrm{mmHg}$, on ventilator settings that include a PEEP $\geq 5 \mathrm{~cm}$ $\mathrm{H}_{2} \mathrm{O}$. And, severe ARDS exists when the $\mathrm{PaO}_{2} / \mathrm{FiO}_{2}$ is $\leq 100 \mathrm{mmHg}$ on ventilator settings that include a PEEP $\geq 5 \mathrm{~cm} \mathrm{H}_{2} \mathrm{O}^{1}{ }^{1}$ Adenoviruses are a family of DNA viruses that are most frequently associated with upper respiratory tract syndromes such as pharyngitis or coryza but can also cause pneumonia. A diagnosis can be made by viral culture, direct antigen assay or PCR assay on a nasopharyngeal aspirate or swab, throat swab, sputum sample, or bronchoalveolar lavage fluid..$^{2,3}$ Adenoviral pneumonia in especially immunocompetent adults presents with respiratory illnessthat progresses rapidly to respiratory failure and often requires mechanical ventilation. We report the case of a previously healthy and immunocompetent woman with severe adenovirus pneumonia who developed rapidly progressive respiratory failure requiring mechanical ventilation and who made a successful recovery after being treated with supportive measures.

\section{Case presentation}

Twenty two-years-old, primigravid, 9 weeks pregnant woman was applied to a local hospital complaining of fever and progressive shortness of breath for the last 8 hours. She was initially treated for suspected urinary tract infection. On obstetric examination, intrauterine ex fetus was detected andcurettage was performed. She was transferred to our ICU for respiratory failure and hypoxemia. There were 4 gestations and 3 abortions on her medical history. On her physical examination; body temperature was $36,6^{\circ} \mathrm{C}$, pulse rate was 126 beats per minute and blood pressure was $165 / 98 \mathrm{mmHg}$. Her respiratory rate was 40 breaths per minute with oxygen saturation of $54 \%$ on room air. Her Glasgow Coma Scale (GCS) was 15. On her chest auscultation, there were inspiratory-expiratory coarsecrackls and expiratory rhoncus on both hemithoraxes. Non-invasive mechanical ventilation (NIMV) was applied. We ordered an arterial blood gas $(\mathrm{ABG})$ test with a fraction of inspired oxygen $\left(\mathrm{FiO}_{2}\right)$ of

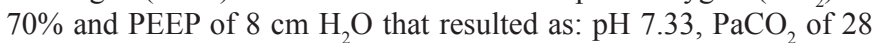
$\mathrm{mmHg}, \mathrm{PaO}_{2}$ of $52 \mathrm{mmHg}, \mathrm{HCO}_{3}$ of $14 \mathrm{meq} / \mathrm{L}$ and $\mathrm{SaO}_{2}$ of $\% 87$. Bilateral infiltrations especially at the middle and lower zones was notable on the chest $\mathrm{x}$-ray (Figure 1). The $\mathrm{PaO}_{2} / \mathrm{FiO}_{2}$ score was $<100$, compatible with severe ARDS. The bedside ECHO showed normal right - left ventricles. She was intubated and connected to protective mechanical ventilation. She was ventilated with pressure controlled synchronous intermittent ventilation (P-SIMV) mode with rate of $14 \mathrm{bpm}$, PEEP of $14 \mathrm{cmH}_{2} \mathrm{O}$, pressure support (PS) of $14 \mathrm{~cm} \mathrm{H}_{2} \mathrm{O}$, $\mathrm{FiO}_{2}$ of 1 . Control ABG was pH:7,39, $\mathrm{PaCO}_{2}$ of $32 \mathrm{mmHg}, \mathrm{PaO}_{2}$ of $80 \mathrm{mmHg}, \mathrm{SaO}_{2}$ of $95 \%$ and $\mathrm{HCO}_{3}$ of $19 \mathrm{meq} / \mathrm{L}$. Oseltamivir $(150$ $\mathrm{mg} / \mathrm{d}$ ) was given by nasogastric tube and intravenous piperacillintazobactam, vancomycin were started as empirical treatment. She was sedated intermittently with propofol, midazolam and remifentanil infusions. Noradrenalin infusion was needed at the initial hours of 
admission, because of a hypotensive episode which did not respond to fluids. Bacterial cultures of blood, urine, and tracheal aspiration were negative. Tracheal aspiration was negative for H1N1 infection but it was positive for adenovirus by polymerase chain reaction (PCR). We administrated $1 \mathrm{mg} / \mathrm{kg}$ /day of methylprednisolone $(60 \mathrm{mg}$ per day) for 4 days and the dose was gradually reduced for 9 days. She was self extubated on the fifth day of admission. She was initiated on NIMVwith an expiratory/inspiratory positive airway pressure of $8 / 13 \mathrm{cmH}_{2} \mathrm{O}$ with a $\mathrm{FiO}_{2}$ of 0.3 and high flow oxygen with nasal canul (Flow: $40 \mathrm{lt} /$ minute and $\mathrm{FiO}_{2}$ of 0,35 ) for one day. She was only continued with high-flow oxygen nasal cannulae (HFNC) for four days. The clinical and radiological improvement was established (Figure 2). The patient was transferred to the department of pulmonary medicine on the tenth day of admission and discharged two days later.

Figure I The chest x-ray on admission.

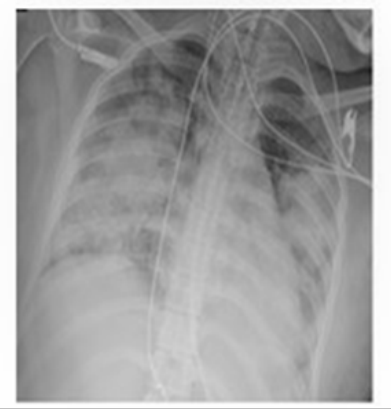

Figure 2 The control chest $\mathrm{x}$-ray.

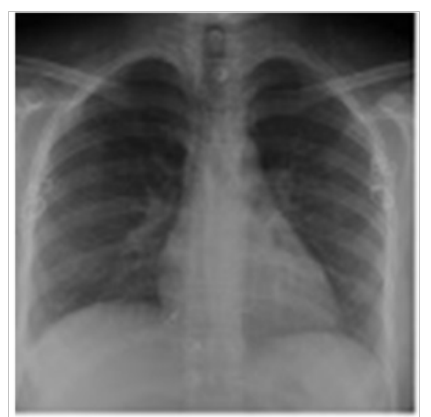

\section{Discussion}

Adenovirus is an uncommon cause of community acquired pneumonia (CAP) (2\%) in non $\square$ military immunocompetent hospitalized adults, and is responsible for $15 \%$ of $\mathrm{CAP}^{4} \mathrm{CAP}$ in immunocompetent adult civilians presents as a non-specific febrile respiratory illness that progresses rapidly to respiratory failure and often requires mechanical ventilation. ${ }^{5}$

Outbreaks of adenovirus infection in long-term nursing facilities and in hospital wards with associated cases of fatal pneumonia have also been described. Respiratory failure developed in five patients. Four patients developed diffuse pulmonary infiltrates and met criteria for ARDS and required high $\mathrm{FiO}_{2}$, PEEP and paralysis to maintain adequate oxygenation. Septic shock developed after the onset of respiratory failure in each patient with ARDS. ${ }^{6}$

For all patients with ARDS, the recommendation is strong for mechanical ventilation using lower tidal volumes $(4-8 \mathrm{ml} / \mathrm{kg}$ predicted body weight) and lower inspiratory pressures (plateau pressure $<30 \mathrm{~cm}$ $\mathrm{H}_{2} \mathrm{O}$ ). For patients with severe ARDS, the recommendation is strong for prone positioning for more than $12 \mathrm{~h} / \mathrm{d}$ ). For patients with moderate or severe ARDS, the recommendation is strong against routine use of high-frequency oscillatory ventilation and conditional for higher positive end-expiratory pressure and recruitment maneuvers. ${ }^{1,7}$ There are no controlled trials and few clinical experience using NIMV to manage patients with ARDS. NIMV may be reserved for the occasional patient with ARDS who is hemodynamically stable, is easily oxygenated, does not need immediate intubation.

In a small $(\mathrm{n}=40)$ trial Zhan et al. randomly assigned patients with ARDS to receive either NIMV or high concentration supplemental oxygen. Patients receiving NIMV were more likely to have improvement of $\mathrm{PaO}_{2} / \mathrm{FiO}_{2}$ and less likely to require intubation (4.8 versus 36.8 percent). ${ }^{8}$ In one trial, 105 patients with acute hypoxemic respiratory failure of varying etiologies were randomly assigned to receive standard medical therapy alone or NIMV plus standard medical therapy. NIMV decreased ICU mortality (18 versus 39 percent) and the intubation rate ( 25 versus 52 percent). ${ }^{9}$

HFNC can oxygenate patients as well as provide a small amount of positive airway pressure and reduce dead space such that it is thought to be a method of delivering noninvasive ventilation. In one meta-analysis of six randomized trials that examined HFNC for the treatment of acute respiratory failure (due to medical conditions [two trials], following extubation [three trials], and during bronchoscopy [1 trial]), HFNC was associated with a lower rate of intubation, when compared with conventional oxygen (12 versus 25 percent) but no difference when compared with NIV (18 versus 23 percent). ${ }^{10}$

\section{Conclusion}

Viral pneumonia should always be considered in differential diagnosis of the etiology of ARDS in young and / or immunosuppressed patients. Appropriate samples for etiological diagnosis should be taken as soon as possible. Early antiviral treatment and protective mechanical ventilation support provide positive results. We present our case to demonstrate the efficacy of protective mechanical ventilation, high PEEP, and high-flow oxygen nasal cannulae in the treatment of severe ARDS due to adenoviral pneumonia.

\section{Acknowledgment}

None.

\section{Conflict of interest}

None declared.

\section{References}

1. Ranieri VM, Rubenfeld GD, Thompson BT, et al. The ARDS Definition Task Force. Acute Respiratory Distress Syndrome: The Berlin Definition. JAMA. 2012;307(23):2526-33.

2. Yang E, Rubin BK. "Childhood" viruses as a cause of pneumonia in adults. Semin Respir Infect. 1995;10(4):232-243.

3. Self WH, Williams DJ, Zhu Y, et al. Respiratory Viral Detection in Children and Adults: Comparing Asymptomatic Controls and Patients With Community-Acquired Pneumonia. J Infect Dis. 2016;213(4):584 591. 
4. Johnstone J, Majumdar SR, Fox JD, et al. Viral Infection in Adults Hospitalized with Community-Acquired Pneumonia Prevalence, Pathogens, and Presentation. Chest. 2008;134(6):1141-1148.

5. Clark TW, Fleet DH, Wiselka JM. Severe community-acquired adenovirus pneumonia in an immunocompetent 44-year-old woman: a case report and review of the literatüre. J Med Case Rep. 2011;5:259.

6. Klinger JR, Sanchez MP, Curtin LA, et al. Multiple cases of lifethreatening adenovirus pneumonia in a mental health care center. Am J Respir Crit Care Med. 1998;157(2):645-649.

7. Fan E, Del Sorbo L, Goligher EC, et al. An Official American Thoracic Society/European Society of Intensive Care Medicine/Society of Critical Care Medicine Clinical Practice Guideline: Mechanical Ventilation in Adult Patients with Acute Respiratory Distress Syndrome. Am J Respir Crit Care Med. 2017;195(9):1253-1263.
8. Zhan Q, Sun B, Liang L, et al. Early use of noninvasive positive pressure ventilation for acute lung injury: a multicenter randomized controlled trial. Crit Care Med. 2012;40:455-460.

9. Brochard L, Mancebo J, Wysocki M, et al. Noninvasive ventilation for acute exacerbations of chronic obstructive pulmonary disease. $N$ Engl J Med. 1995;333(13):817-22.

10. Ou X, Hua Y, Liu J, et al. Effect of high-flow nasal cannula oxygen therapy in adults with acute hypoxemic respiratory failure: a metaanalysis of randomized controlled trials. CMAJ. 2017;189(7):E260E267. 